\title{
Tomasz Szybisty
}

ORCID: 0000-0002-6047-5716

Pädagogische Universität Kraków, Kraków

DOI: $10.19195 / 0435-5865.144 .2$

\section{Die Kraft der Gletscher. Ein Beitrag zur politischen Ikonographie in der Literatur der Napoleonischen Kriege}

\begin{abstract}
s
Der vorliegende Aufsatz widmet sich den politisch-nationalen Auslegungen der eisigen Landschaft in ausgewählten literarischen Werken der Napoleonischen Zeit. Eingangs wird die Aufwertung der polaren und alpinen Regionen im 17. und 18. Jahrhundert umrissen, in deren Folge die bis dahin negativ konnotierten Gebiete als antizivilisatorische Sehnsuchts- oder Erkenntnisorte umgedeutet wurden. Einen Höhepunkt fand diese Entwicklung am Anfang des 19. Jahrhunderts. Der hohe Norden sowie die Alpen als Zitat des Nordischen wurden insbesondere zur Zeit der Befreiungskriege in der politischen Lyrik als Symbole der Freiheit und Hoffnung eingesetzt, da diese Himmelsrichtung assoziativ u.a. mit der vermuteten Herkunft der Germanen aus Skandinavien und der Niederlage Napoleons im winterlichen Russland verbunden wurde.
\end{abstract}

Schlüsselwörter: Norden, Alpen, Lyrik, Politik, Napoleonische Zeit

\section{The power of glaciers: Shedding additional light on the political iconography found in the lyrical production of the Napoleonic era}

This article undertakes to investigate national-political connotations attendant to glacier-featuring landscapes in some literary works of the Napoleonic era. The point of departure for this analysis is marked by the outline of the 18th-century re-evaluative process which concerned the cultural conventional wisdom vis-à-vis the symbolic significance of polar and high-elevation regions. In that time, they came to be regarded as off-the-beaten-track refuges and loci affording illumination. This new trend found its culmination at the beginning of the 19th century. The Far North (as well as the Alps) may have functioned in that period as a symbol connoting freedom and hope for a vic- 
tory over the French; this sentiment achieved particular prominence in the lyrical production of the Napoleonic period. The German national psyche invested such regions i.a. with two qualities: firstly, they were reminiscent of the cradle of the Germanic people, and secondly, they invoked the debacle of Napoleon's winter military campaign in Russia.

Keywords: the North, the Alps, lyrical literature, politics, the Napoleonic era

Tomasz Szybisty, Instytut Neofilologii, Uniwersytet Pedagogiczny w Krakowie, ul. Studencka 5, 31-116 Kraków, Polen, E-Mail: tomasz.szybisty@gmail.com

Received: 26.09.2018, accepted: 8.04.2019

In der imaginären Kulturgeographie Europas haftete den lebensfeindlichen, eisigen Landschaften bis in die Neuzeit hinein hauptsächlich ein negativer Charakter an. In John Miltons eindrücklicher Beschreibung der Hölle, die sich in seinem Verlorenen Paradies (1667) findet, heißt es etwa:

Jenseits des Stroms liegt ein gefrornes Land,

Wild, finster, stets von Stürmen, Wirbelwinden

Und Hagelwettern heimgesucht; nie taut

Auf festem Lande dieser Hagel auf;

$\mathrm{Zu}$ Haufen türmt er sich und scheint von fern

Verfallenes Gemäuer; [...]

Des Todes Schattenreich; - Tod überall!

Ein Weltall voller Tod, das Gott im Fluch

Erschaffen; böse, nur fürs Böse gut;

Wo alles Leben stirbt, wo Tod nur lebt,

Und die Natur verwahrlost, Ungeheuer,

Scheusale nur und Mißgeburten heckt,

Viel ärger als die Fabel sie ersann. (Milton o.J.: 53-54)

Diese negative, von der Antike überkommene Vorstellung büßte im 18. Jahrhundert weitgehend ihre Vorherrschaft ein. Die Umwertung ging einerseits auf die Erkundung und Erschließung von polaren und alpinen Regionen infolge wissenschaftlicher Expeditionen zurück; andererseits war sie auch durch die Philosophie der Aufklärung mit ihrer Forderung „Zurück zur Natur!“ und später durch den romantischen Pantheismus bedingt, die beide von Menschenhand unberührte Gegenden als antizivilisatorische Sehnsuchts- und Erkenntnisorte einstuften (s. Woźniakowski 1974, Wilson 2003). Die Alpen und der hohe Norden eigneten sich umso besser als „Labore“, in denen man die Geheimnisse der menschlichen Seele und des Weltalls ergründen konnte, als sie durch Gletscher und andere Eisformationen geprägt waren. Da man die Kristallisation des Wassers als das Vorbild der Kristallisation per se betrachtete (Ritter 1805: 261) und in diesem Prozess gelegentlich einen physikalischen Vorgang vermutete, der sowohl der organischen als auch anorganischen Natur zugrunde liegen sollte (vgl. Köchy 1997: 128-139), 
könnte die Aufwertung der „kristallinen“ Gebiete zum Teil auch auf die naturwissenschaftlichen Theorien der Zeit zurückzuführen sein.

Nicht außer Acht zu lassen sind zudem die allmähliche Relativierung der klassischen Ästhetik und die damit einhergehende Infragestellung der bis dahin gültigen Kulturmuster, die auf der römisch-griechischen Antike basierten. Die Umwälzungen auf diesem Gebiet manifestierten sich insbesondere im intensiv diskutierten Konzept des Sublimen bzw. Erhabenen, dessen neuzeitliche Wurzeln auf die von Nicolas Boileau-Despréaux initiierte Diskussion über die Longinos zugeschriebene Schrift Perì hýpsous zurückgehen. Folgenschwer war in diesem Kontext allerdings auch eine persönliche Erfahrung des britischen Kritikers und Dramatikers John Dennis, die er machte, als er auf einer Reise nach Italien im Herbst 1688 die Alpen überquerte (vgl. u.a. Zelle 1989: 55-73). Seine Eindrücke bei der gefährlichsten Wegetappe bezeichnete er in einem Brief als „herrliches Grausen“ (delightful Horror) und „schreckliche Freude“ (terrible Joy) (Dennis 1943: 380). Einige Jahre später setzte er sich mit diesem Gefühl, das sich aus Bewunderung und Schrecken zusammensetzt, philosophisch auseinander und legte mit seiner Schrift The Grounds of Criticism in Poetry die Grundlagen für die Theorie des Erhabenen (Reif 2010: 167). Als „erhaben“ bezeichnete Burke in seinen vielgelesenen Philosophischen Untersuchungen von 1757 diejenigen Phänomene, die gefährlich und zugleich anziehend wirken, und zählte unter anderem Dunkelheit, Kraft und Unendlichkeit zu ihren Eigenschaften. Der englische Philosoph bezog diese Kategorie vor allem auf natürliche Erscheinungen (Burke 1772). In Burkes Fußstapfen trat kurz darauf Immanuel Kant, für den die Nacht, der „Anblick eines Gebirges, dessen beschneite Gipfel sich über Wolken erheben, die Beschreibung eines rasenden Sturmes, oder die Schilderung des höllischen Reiches von Milton“ erhaben, der Tag und „die Aussicht auf blumenreiche Wiesen, Thäler mit schlängelnden Bächen, bedeckt von weidenden Heerden“" (Kant 1867: 230) jedoch schön waren. Die Kategorie des Erhabenen war also ein probates Mittel, um die eisigen, alpinen oder nördlichen Landschaften aufzuwerten.

Eine katalytische Rolle in diesem Prozess spielte der in ganz Europa rezipierte Ossian, ,das poetische Paradigma des Erhabenen in der zweiten Hälfte des 18. Jahrhunderts" (Schmidt 2003: 350). Das 1760 veröffentlichte Epos, dessen vermeintlich altschottischer Autor als Homer des Nordens apostrophiert wurde, gilt als Meilenstein in der Entdeckung der Vor- und Frühgeschichte und des Mittelalters, aber auch des Volkstümlichen, in dem man den reinen Urgeist der Nation zu finden glaubte. Ossian trug zudem maßgeblich zur Popularität der rauen, nordischen Gebiete bei, die durch Berge, Felsen, extreme Wetterereignisse, Schnee, Eis, Nebel und dichte Wälder bestimmt sind. In der deutschen Literatur der Sturmund-Drang-Zeit wird die neue Landschaftsästhetik deutlich spürbar, wenn Goethes Werther ausruft: 
Ossian hat in meinem Herzen den Homer verdrängt. Welch eine Welt, in die der Herrliche mich führt! $\mathrm{Zu}$ wandern über die Heide, umsaust vom Sturmwinde, der in dampfenden Nebeln die Geister im dämmernden Lichte des Mondes hinführt. Zu hören vom Gebirge her im Gebrülle des Waldstroms halb verwehtes Ächzen der Geister aus ihren Höhlen, und die Wehklagen des zu Tode gejammerten Mädgens, um die vier moosbedeckten, grasbewachsenen Steine des edelgefallnen ihres Geliebten. (Goethe 1998: 71)

Wolf Gerhard Schmidt geht sogar so weit, von einem locus ossianicus zu sprechen, der um 1800 weitgehend konventionalisiert worden sei und als Folie für Naturbetrachtungen dienen konnte (Schmidt 2003: 350). So schreibt Johanna Schopenhauer in ihren Erinnerungen von einer Reise in den Jahren 1803, 1804 und 1805:

Es war ein Ossianscher Tag, graue Nebel hiengen an den Spitzen der Berge, wogten zuweilen herab und durcheilten, gejagt vom Winde, wie Geistergestalten die Schluchten der Felsen. Einzelne Sonnenblicke flogen über das Thal, durch welches bald silberhell, bald wild tobend ein starker Bach sich wand. [...] Hier erscheint die Natur, wie Ossian sie malte, die Ströme, die Felsen, die uralten einzelnen Eichen. (Schopenhauer 1813: 157)

In ihren nächsten Sätzen kommt zum ästhetischen, wie übrigens auch in Werther, der historische Diskurs hinzu:

Der Wind heult über die Haide, die Distel wiegt ihr Haupt im Sturme am Grabe der alten Krieger. Die vier grauen, bemoosten Steine erheben sich noch einsam am Hügel der Helden und verkünden stumm dem stillen Wanderer die Geschichte vergangener Jahrhunderte. (Schopenhauer 1813: 157-158)

Der locus ossianicus war nie ausschließlich ein locus naturalis. Vielmehr enthielt er immanent zugleich die Assoziation mit einer bestimmten Kulturformation, dem Europa der nordischen Völker, die sich von den Südeuropäern nicht nur durch ihre Sitten, sondern auch, so glaubte man, durch physische und geistige Veranlagungen unterschieden hätten (Meyer 2003: 334-344). Insofern ging es nicht allein um eine geographisch, sondern ebenso kulturell bzw. imaginativ definierte Region, denn der Norden ist - wie Sophie Dietrich feststellte - ein „Konglomerat aus unterschiedlichen Vorstellungen“, die immer „vom eigenen Standpunkt“ abhingen (Dietrich 2014: 8). Je nach Land erhielt diese Assoziation eine andere Färbung: eine keltische in Frankreich und Großbritannien, eine germanische in den deutschsprachigen Ländern. Dabei wurde das Konzept des Nordischen mit der Zeit immer häufiger für politische Zwecke instrumentalisiert.

Die politisch-nationale Dimension der nordischen Landschaft, die meistens nur mit einigen wenigen typisierten Requisiten evoziert wird, kommt bereits in vielen lyrischen Werken des letzten Viertels des 18. Jahrhunderts zum Ausdruck, etwa im 1788 veröffentlichten Gedicht Das Hünengrab von Ludwig Theobul Kosegarten:

[...] Bei den Edelgefallnen!

Bei der Eiche und dem Stein! Ich schwöre der Ahnentugend! 
Schwöre dir, Treue der Väter!

Schwöre dir, Keuschheit der Ahnen!

Schwöre dem Ausland hohen Troz!

Schwöre dir, Biedersinn und Opferdurst für die Brüder!

Wie das Moos duftet! Wie die Eiche rauscht!

Wie die Unke läutet und die Nachtigalen flöten!

Der Mond tanzet in lichten Wolken,

und die thauschimmernde Saat schwimt silbern den Hügel hinan!

(Kosegarten 1788: 387-388)

Die bemoosten Steine, unter denen die germanischen Ahnen des lyrischen Ichs ruhen, sind hier die Quelle der moralischen und nationalen Kräfte. Bei der Schilderung der nächtlichen Landschaft klingen sowohl Ossian als auch eine stimmungsevokative Friedhofslyrik an. Ihre Beschreibung beschränkt sich auf rudimentäre, zeichenhafte Elemente, wie uralte Grabsteine und Eichen, die an den Norden erinnern. Sie bilden die Szenerie, in der ein pathetischer Eid abgelegt werden kann.

Neben vielen anderen patriotischen Figuren und Symbolen (Hermand 1995: 14) begegnet diese Motivik verstärkt in der Ära der Napoleonischen Kriege. ${ }^{1}$ So ruft Ernst Moritz Arndt in seinem Lied Auf die Schlacht bei Groß-Görschen oder Lützen dazu auf, den Gefallenen ein „deutsches“ Denkmal zu errichten, das an ein Hünengrab erinnert:

Auf! und thürmt den Berg von Steinen!

Thürmt ein Deutsches Heldenmal!

Sonne, komm' mit hellsten Scheinen,

Leuchte drauf den schönen Strahl

Auf! und pflanzet grüner Eichen

Ernste Haine ringumher!

Betet, daß in Deutschen Reichen

Buben freveln nimmermehr!

(Arndt 1814a: 89)

Die eisigen, frostigen Winterlandschaften, die als Höhepunkte der nordischen Szenerie gelten können, wurden zu dieser Zeit vermehrt politisch und patriotisch interpretiert. Diese Tendenz zeichnete sich insbesondere nach dem gescheiterten Russlandfeldzug Napoleons im Spätherbst und Winter 1812 ab. In Arndts Gottes Gericht wird die winterliche Natur zum eschatologischen Schauplatz der Katastrophe der Grande Armée (Arndt 1814b: 30-31). Dasselbe Bild ist auch im Weihgesang zum Verderben eines anonymen Autors zu finden:

Bezwinger, dein Maaß war voll,

Der Rache Donner erscholl -

Wehe dir! Weh

Auf Nordlands Schnee.

${ }^{1}$ Zur politischen Lyrik dieser Zeit, insb. der Befreiungskriege s. v.a. Weber (1991); dort weiterführende Literatur. 
$[\ldots]$

Mit der Vernichtung Gewalt

Auf den beeisten Gefilden

Hat dich die Rache ereilt!

(Anonym 1813: 15)

In der Lyrik der Befreiungskriege konnte der Norden als Verweis auf die Urheimat der Germanen und die symbolische Quelle der nationalen Hoffnung fungieren. Nach dem misslungenen Feldzug Napoleons gegen Moskau im Jahr 1812 und dem Zusammenbruch der "Großen Armee“, die durch den frostigen Winter ebenso wie durch die zaristischen Soldaten dezimiert wurde, konnte der Norden allerdings auch als Metonymie für Russland verwendet werden, da in der geistigen Kartographie Europas das Land sich bis in die Mitte des 19. Jahrhunderts nicht im Osten, sondern im Norden befand (Hildermeier 2013: 1316). Die beiden Bedeutungsfacetten der nordischen Himmelsrichtung fallen im Gedicht Germanias Rückkehr von Johann Peter von Hornthal, das er 1814 dem Zaren Alexander I. widmete, zusammen. Die titelgebende Germania sucht vorübergehend Zuflucht im Norden, wo sie die Freiheit „für schön're Zeit" wahrt, um nach Napoleons russischer Niederlage, ,[u]mgeben rings von mächt'ger Heldenschar“, ,zum freien Vaterlande, / Zum alten Heldenvolk“ (Hornthal 1814: 1138) zurückzukehren. Sie schreitet gegen den Rhein, und die letzte Etappe ihres siegreichen Zuges wird durch das Hissen „der Freiheit Fahnen“ am Kölner Dom markiert. Neben dem Meisterwerk der gotischen Architektur, deren Ursprünge schon in der Renaissance mit den Germanen bzw. Deutschen verbunden wurden (vgl. u.a. Brandis 2002), und einem alten Barden, der „den Schlachtensang“ (Hornthal 1814: 1138) singt, evoziert Hornthal die germanische Tradition der Deutschen auch durch den Rekurs auf den „heil'ge[n] Hermannskrieg“ (Hornthal 1814: 1138). In dieser Perspektive sind die Franzosen eindeutig als Nachkommen der Römer zu betrachten, die in der Schlacht im Teutoburger Wald von Arminius eine Niederlage erlitten haben und die es nun abermals zu besiegen gilt - eine Figur, der sich einige Jahre zuvor auch Heinrich von Kleist bediente (siehe unten).

In einigen weiteren patriotisch und sogar chauvinistisch gesinnten Gedichten der Zeit wurde der Norden zum Hort der Freiheit stilisiert. So schrieb 1813 Theodor Körner in seinem Aufruf: „Hell aus dem Norden bricht der Freiheit Licht“, und appellierte zugleich an „,das alte Volk des Siegs“, „,den Stahl in Feindes Herzen [zu] tauchen" (Körner 1814: 37-39). Auch Gustav Scholz forderte 1814 die von Napoleon unterdrückten Völker auf, den Tyrannen umzustürzen. Dabei sollten sie ihre Hoffnung aus dem kalten Norden schöpfen: „Nach Norden schauet: aus klingendem Eis / entkeimte der Freyheit grünendes Reis“"(Scholz 1814: 130).

Die Symbolik des Nordens, die sich in den zitierten, politisch motivierten Texten manifestiert, war das Ergebnis des bereits oben erwähnten, im 18. Jahrhundert begonnenen Umwertungsprozesses, der um 1800 in einen regelrechten Nordlandmythos mündete. Einer der Kerngedanken dieses Mythos, der von Her- 
der, in erster Linie durch seinen Aufsatz Iduna, oder der Apfel der Verjüngung (1796), angeregt und von der romantischen Bewegung aufgenommen wurde, war die Überzeugung, dass das sagenumwobene skandinavische Mittelalter, dessen literarisches Erbe immer häufiger gelesen und bearbeitet wurde, eine Quelle des genuinen Germanentums darstelle und ein wichtiger Baustein für die Bildung der deutschen Nation sei. Zugleich wurde der Norden in der Literatur der Frühromantik oft mit „magnetische[n] und Astralbilder[n]“ (Bohrer 1961: 25) verbunden, was ihn ,als ein geheimnisvolles Kräftezentrum oder die Repräsentanz ideeller Inhalte“ (Bohrer 1961: 25-26), und im politischen Kontext „als geschichtsmächtiger, geschichtserlösender Weltgegend" (Bohrer 1961: 63) erscheinen ließ. Der Bedeutungswandel schloss die alte, negative Wahrnehmung der polaren und alpinen Gebiete allerdings nicht komplett aus. Nach wie vor begegnet sie in vielen literarischen Werken der Zeit und wurde ebenfalls politisch eingesetzt.

Der Konnex von eisigen Landschaften und patriotischen Inhalten erreichte am Anfang des 19. Jahrhunderts in Heinrich von Kleists Ode Germania an ihre Kinder seinen Höhepunkt (erste Fassung 1809²). Hier sind es die Alpen, die als Zitat des Nordischen herangezogen und als Quelle der nationalen Kraft ausgewiesen werden:

3

Wie der Schnee aus Felsenrissen:

Wenn auf ew'ger Alpen Höhn, Unter Frühlings heißen Küssen,

Siedend auf die Gletscher gehen:

Katarakten stürzen nieder,

Fels und Wald folgt ihrer Bahn,

Das Gebirg' hallt donnernd wieder,

Fluren sind ein Ocean.

Chor.

So verlaßt, voran der Kaiser,

Eure Hütten, eure Häuser,

Schäumt ein uferloses Meer,

Über diese Franken her. ${ }^{3}$ (Kleist 1990: 428)

In Kleists dialogisch aufgebautem Gedicht, das sich nach dem Erstdruck 1813 zur Zeit der Befreiungskriege einer ungemein großen Popularität erfreute (Müller-Salget 1990: 998), um noch nach 1918 ,immer wieder in den Dienst des Revanchismus gegenüber dem französischen ,Erbfeind" gestellt“" (Müller-Salget 1990: 1002) zu werden, ruft Germania die Volksstämme Deutschlands zusammen, um sie im blutigen Kampf gegen Napoleon zu vereinen. Die Deutschen werden

2 Über die verschiedenen Fassungen der Ode (bislang sind neun bekannt) s. Müller-Salget (1990: 999-1001).

${ }^{3}$ Die hier zitierte Version der Ode bezeichnet Müller-Salget als „Version a“. Dabei handelt es sich wahrscheinlich um das 1809 an Heinrich Joseph von Collin geschickte Manuskript (Müller-Salget 1990: 998). 
aufgefordert, ein Meer zu bilden, das die neuen Römer - die verhassten Franzosen - vom Angesicht der Erde hinwegschwemmen möge. In der Ikonographie der Ode hat dieses Meer seinen Ursprung in den alpinen Gletschern. Das von Kleist gewählte Bild der Schneeschmelze wertet Walter Hettche unter Berufung auf Hans Wolf Jäger als ,motivisches Versatzstück, dem man in politischer Lyrik aller Epochen immer wieder begegnet" (Hettche 1990: 167). Neben dem allgemeinen Charakter ist die Schneeschmelze-Metaphorik in dem Gedicht ebenfalls vor dem Hintergrund der Aufwertung der eisigen Gebiete zu sehen, die zu dieser Zeit assoziativ mit dem Germanischen, dem „Urdeutschen“, verbunden wurden. Der wohl prägnanteste Ausdruck dieser Verbindung im Schrifttum des frühen 19. Jahrhunderts findet sich in Joseph Görres' Aufsatz Religion in der Geschichte, der bereits 1807, also zwei Jahre vor der Entstehung der Germania, erschien. Görres bediente sich darin der Bildmetapher der „,nordischen Gletscher“, die in der Völkerwanderungszeit gegen das kraftlose Römische Reich angedrängt hätten (Görres 1807: 349).

Die „Alpen Höhn“, die in Kleists Ode als Quelle nationaler Bestrebungen fungieren, ermöglichen noch eine andere Lesart. Man kann die Synekdoche als Reminiszenz an den Schweizer Mythos ${ }^{4}$ deuten, dessen wesentliche Bestandteile „das Naturerhabene der Alpenlandschaft, die natürliche Einfalt und patriarchalische Lebensführung, die Landlebenidylle" und nicht zuletzt „der Freiheitswille der Schweizer“ (Reif 2010: 170) waren.

Wie nachhaltig sich die politisch gefärbte Vorstellung von der kalten, nordischen Landschaft als Ort ursprünglicher Kräfte und staatlicher Erneuerung sogar über Napoleons Sturz hinaus durchgesetzt hat, zeigt die fragmentarische Erzählung Die Königin von Borneo des preußischen Kronprinzen Friedrich Wilhelm. 1997 erstmals publiziert, findet sich die Geschichte in einer Reihe von Briefen, die der künftige König vom September 1816 bis zum Sommer 1817 an seine Schwester Charlotte geschrieben hat. ${ }^{5}$ Die Handlung der Erzählung nimmt ihren Lauf kurz nach dem Einzug der alliierten Truppen in Paris 1814. Der Protagonist ist Friedrich Wilhelm selbst. Er wird von Magdalene, einer Abgesandten der Prinzessin Satischeh-Cara von Borneo, überredet, sich in das exotische Reich zu begeben, um dort Taufpate der Prinzessin zu sein. Satischeh-Cara war für das Christentum gewonnen worden, als sie „einst, vor vier Jahren, von jugendlicher Wißbegierde getrieben, verlangt habe, die Glaubensgenossen ihrer Lieblingsgespielinn (der Magdalene selbst) kennen zu lernen, die in hartem Druck die ungeheuren Gebirge der Insel mit den Menschenfressern theilten“ (Friedrich Wilhelm 1997: 29). Die Prinzessin hatte ihren Vater, König Rußang-Gehun, gebeten, bei einer Jagd „die Christen in den Bergen aufzusuchen" (Friedrich Wilhelm 1997: 29). Erst nach neun Tagen wurde die Gemeinde gefunden, da sie zurückgezogen in einem para-

${ }^{4}$ Zum Schweizer Mythos s. u.a. Ziehen (1922) und Gruenter (1994).

${ }^{5}$ Zur Entstehungsgeschichte und Interpretation dieses Textes s. v.a. Gersdorff (1997: 9-20), Kroll (1997: 117-138), Polaschegg (2005: 450-530), Johannsen (2007: insb. 63-95). 
diesischen Tal wohnte, ${ }^{6}$ jenseits eines „erstarrten Rücken[s] der Berge“ (Friedrich Wilhelm 1997: 30) und „unfern der Schneegrenze“ (Friedrich Wilhelm 1997: 29). Es stellte sich heraus, dass die Gemeinde noch zu der „durch den Apostel Thomas in Indien gestifteten Kirche“ (Friedrich Wilhelm 1997: 30) gehörte. Der König, der sich als ,einer auf der Jagd Verirrter“ ausgegeben hatte, wurde von den Christen „mit dem Liebeseifer der ersten Kirche aufgenommen und gepflegt" (Friedrich Wilhelm 1997: 30). Diese Erfahrung bewog ihn und seine Tochter zur Konversion zum Christentum. Nachdem Friedrich Wilhelm diese Geschichte gehört hat, zögert er nicht lange und erklärt sich dazu bereit, die Reise nach Borneo anzutreten und die Patenschaft für die Prinzessin zu übernehmen. Nach seiner Ankunft wird der beabsichtigte Glaubenswechsel des Königs am Tag vor der Taufe den versammelten, bislang unwissenden bornesischen Fürsten mitgeteilt. Brunninghir, König von Banjer-Maßin, stellt sich gegen das Vorhaben und zettelt in der Nacht einen Revolutionsversuch an, der von den entmachteten Brahmanen unterstützt wird. Die Rebellen werden besiegt, wozu Friedrich Wilhelm maßgeblich beiträgt. So kann am folgenden Tag die Taufe des Herrscherhauses und Tausender Untertanen feierlich vollzogen werden. Kurz darauf bricht die Geschichte abrupt ab.

Rolf H. Johannsen hat überzeugend gezeigt, dass die Erzählung, die auf den ersten Blick lediglich wie eine orientalische Phantasie anmutet, unter Hinzuziehung von Briefen des Kronprinzen aus den Befreiungskriegen auch als Allegorie gelesen werden kann (Johannsen 2007: 83-86). Die Königin von Borneo liefert darüber hinaus einen Einblick in die Vorstellungen Friedrich Wilhelms zur Legitimität eines Herrscherhauses. So ist das politische System des bornesischen Staates „ungefähr [...] wie im Heiligen Römischen Reich“ (Friedrich Wilhelm 1997: 68), und eben ein solches ausgewogenes Herrschaftsgefüge ersehnte sich der junge Thronfolger in einem Brief auch für Europa (Johannsen 2007: 84). Es gibt zudem Bezüge zum besiegten und verbannten Kaiser der Franzosen: die Tafeln für das Gefolge der bornesischen Könige und Fürsten bei dem feierlichen Mahl am Vortag der Taufe schildert der Kronprinz prächtiger als „Napoleons Staatstafel“ (Friedrich Wilhelm 1997: 90; vgl. Johannsen 2007: 82). Der von den Aufständischen getragene wandelnde Turm, ,auf welchem Indras Bildsäule saß“ (Friedrich Wilhelm 1997: 95) und der unter Führung des preußischen Gastes zerstört wird, geht, so Johannsen, auf den von Friedrich Wilhelm in einem Brief an Charlotte erwähnten Versuch der Pariser Bevölkerung zurück, die Statue Napoleons von der Siegessäule auf der Place Vendôme zu stürzen (Johannsen 2007: 85). Brunninghir ist wie Napoleon ein Usurpator, der schlussendlich von einem legitimen Herrscher besiegt wird. Der Machtanspruch des amtierenden Königs Rußang-Gehun basiert dagegen auf der Anciennität einer Dynastie, die mythologische Ursprünge vorzuweisen hat, und der langen Tradition seines neuen Bekenntnisses, das insofern

${ }^{6}$ Andrea Polaschegg (2005: 501) sieht im Entwurf des natürlichen und idyllischen Lebensraumes der bornesischen Christen „deutliche Anleihen“ bei der Erzählung Paul und Virginie von Jacques-Henri Bernardin de Saint Pierre, einer der Lieblingslektüren des Kronprinzen. 
wahr und unverdorben ist, als es sich direkt von einem Apostel herleitet (vgl. Polaschegg 2005: 492-506; Johannsen 2007: 78-82). Diese Religion, die nun zur Erneuerung des bornesischen Staates beiträgt, hat über Jahrhunderte hinweg in einem bergischen Refugium, „unweit der Schneegrenze“, überlebt. Im Kontext der hier angestellten Überlegungen scheint dieser Ausdruck, neben anderen von Johannsen und Polaschegg in der Erzählung gefundenen Bezügen, ein aktueller Reflex auf die symbolische Aufladung der nordischen Landschaften zu sein.

In der veränderten politischen Situation nach dem Wiener Kongress büßte die nordisch-germanische Thematik ihre Bedeutung im politisch-nationalen Sinn immer mehr ein, um am Ende des Jahrhunderts mit dem erwachenden Interesse am Germanentum erneut aufzublühen und im „Germanenwahn“ des Nationalsozialismus ein unrühmliches Ende zu finden. Vor diesem Hintergrund kann die Popularität des nordischen Themenbereichs in Deutschland über die Zeiten hinweg zu einem gewissen $\mathrm{Ma} ß$ als ein literarischer Indikator nationaler und ethnozentrischer Denkmuster angesehen werden. Unter vielen literarischen Bildern und Stoffen, die den Fundus für das nordische Motiv bilden, kam der eisigen Landschaft - was hier ansatzweise gezeigt werden konnte - eine nicht unerhebliche Bedeutung zu.

\section{Literatur}

Anonym (1813): Weihgesang zum Verderben. In: Kriegslieder der Teutschen. Zum Besten der Errichtung der Königl. Preuß. Schwarzen Freiwilligen-Schaar. Teutschland [Berlin]. S. 15-17.

Arndt, Ernst Moritz (1814a): Auf die Schlacht bei Groß-Görschen oder Lützen, den 2ten May 1813. In: Ergießungen Deutschen Gefühles in Gesängen und Liedern bey den Ereignissen dieser Zeit. o.O. S. 87-90.

Arndt, Ernst Moritz (1814b): Gottes Gericht. In: Ders.: Lob teutscher Helden. [Frankfurt am Main]. S. 30-31.

Bohrer, Karl-Heinz (1961): Der Mythos vom Norden. Studien zur romantischen Geschichtsprophetie. Köln.

Brandis, Markus (2002): La maniera tedesca. Eine Studie zum historischen Verständnis der Gotik in Italien der Renaissance in Geschichtsschreibung, Kunsttheorie und Baupraxis. Weimar.

Burke, Edmund (1772): Philosophische Untersuchungen über den Ursprung unsrer Begriffe vom Erhabnen und Schönen. Übers. v. Christian Garve. Riga.

Dennis, John (1943): Letter describing his crossing the Alps, dated from Turin, Oct. 25, 1688. In: Ders.: The Critical Works, Bd. 2. Hg. v. Edward Niles Hooker. Baltimore. S. 380-382.

Dietrich, Sophie (2014): Der nordische Naturraum und das Erhabene: Eine Fallstudie. In: Ecozona. European Journal of Literature, Culture and Environment 5, Nr. 2, S. 6-22.

Friedrich Wilhelm IV. (1997): Die Königin von Borneo. Ein Roman. Hg. v. Frank-Lothar Kroll. Berlin.

Gersdorff, Dagmar von (1997): Von Berlin bis Borneo. In: Friedrich Wilhelm IV., Die Königin von Borneo. Ein Roman. Hg. v. Frank-Lothar Kroll. Berlin. S. 9-20.

Goethe, Johann Wolfgang von (1998): Die Leiden des jungen Werthers. In: Ders.: Werke. Jubiläumsausgabe. Hg. v. Friedmar Apel et al. Bd. 4. Hg. v. Wilhelm Voßkamp, Waltraud Wiethölter. Frankfurt am Main/Leipzig. S. 7-102. 
Görres, Joseph (1807): Religion in der Geschichte. In: Daub, Carl / Creuzer, Freidrich (Hrsg.): Studien. Jahrgang 1807. Heidelberg. S. 313-480.

Gruenter, Rainer (1994): Der Mythos der Schweiz im 18. Jahrhundert. Ein Landschaftsbild. In: Thomke, Hellmut / Bircher, Martin / Proß, Wolfgang (Hrsg.): Helvetien und Deutschland. Kulturelle Beziehungen zwischen der Schweiz und Deutschland in der Zeit von 1770-1830. Amsterdam-Atlanta, GA. S. 7-19.

Hafner, Ulrike (1996): „Norden” und „Nation” um 1800. Der Einfluß skandinavischer Geschichtsmythen und Volksmentalitäten auf deutschsprachige Schriftsteller zwischen Aufklärung und Romantik (1740-1820). Trieste.

Hermand, Jost (1995): Die gescheiterte Hoffnung. Zur Malerei der Befreiungskriege. In: Ders.: Avantgarde und Regression. 200 Jahre deutsche Kunst. Leipzig.

Hettche, Walter (1990): Heinrich von Kleists Lyrik. Frankfurt am Main/Bern/New York (= Europäische Hochschulschriften, Reihe I: Deutsche Sprache und Literatur, Bd. 859).

Hildermeier, Manfred (2013): Geschichte Russlands. Vom Mittelalter bis zur Oktoberrevolution. München.

Hornthal, Johann Peter (1814): Germania’s Rückkehr. In: Zeitung für die Elegante Welt 143 (21.07.1814), S. 1137-1139.

Johannsen, Rolf H. (2007): Friedrich Wilhelm IV von Preußen. Von Borneo nach Rom. Sanssouci und die Residenzprojekte 1814 bis 1848 . Kiel.

Kant, Immanuel (1867): Beobachtungen über das Gefühl des Schönen und Erhabenen. In: Ders.: Sämtliche Werke. In chronologischer Reihenfolge. Hg. v. G. [ustav] Hartenstein. Bd. 2. Leipzig. S. 227-280.

Kleist, Heinrich von (1990): Germania an Ihre Kinder. Eine Ode. In: Ders.: Sämtliche Werke und Briefe in vier Bänden. Hg. v. Klaus Müller-Salget. Bd. 3: Erzählungen / Anekdoten / Gedichte / Schriften. Frankfurt am Main. S. 426-432.

Köchy, Kristian (1997): Ganzheit und Wissenschaft. Das historische Fallbeispiel der romantischen Naturforschung. Würzburg (= Epistemata. Reihe Philosophie, Bd. 180).

Körner, Theodor (1814): Aufruf. In: Ders.: Leyer und Schwerdt. Berlin. S. 37-39.

Kosegarten, Ludwig Theobul (1788): Das Hünengrab. In: Ders.: Gedichte. Bd. 1. Leipzig. S. 384-388.

Kroll, Frank-Lothar (1997): Friedrich Wilhelm IV. als Dichter. Über das Romanfragment „Die Königin von Borneo “. In: Friedrich Wilhelm IV., Die Königin von Borneo. Ein Roman. Hg. v. Frank-Lothar Kroll. Berlin. S. 117-138.

Meyer, Silke (2003): Die Ikonographie der Nation. Nationalstereotype in der englischen Druckgraphik des 18. Jahrhunderts. Münster/New York/München/Berlin.

Milton, John (o.J.): Das verlorene Paradies. Übers. v. Samuel Gottlieb Bürde. Halle a.d.S.

Müller-Salget, Klaus (1990): Kommentar. In: Heinrich von Kleist, Sämtliche Werke und Briefe in vier Bänden. Hg. v. Klaus Müller-Salget. Bd. 3: Erzählungen / Anekdoten / Gedichte / Schriften. Frankfurt am Main. S. 675-1236.

Polaschegg, Andrea (2005): Der andere Orientalismus. Regeln deutsch-morgenländischer Imagination im 19. Jahrhundert. Berlin-New York (= Quellen und Forschungen zur Literatur- und Kulturgeschichte. Hg. v. Ernst Osterkamp, Werner Röcke, 35 (269)).

Reif, Wolfgang (2010): Die Alpen und die Schweiz in Literatur und Kunst von der Aufklärungszeit bis zur klassisch-romantischen Epoche. In: Faber, Richard / Holste, Christine (Hrsg.): Arkadische Kulturlandschaft und Gartenkunst. Eine Tour d'Horizon. Würzburg. S. 167-199.

Ritter, Johann Wilhelm (1805): Das Electrische System der Körper. Leipzig.

Schmidt, Wolf Gerhard (2003): „Homer des Nordens “ und „Mutter der Romantik“. James Macphersons Ossian und seine Rezeption in der deutschsprachigen Literatur. Bd. 1: James Macphersons Ossian, zeitgenössische Diskurse und die Frühphase der deutschen Rezeption. Berlin/New York.

Scholz, Gustav (1814): Aufruf an die Söhne des Vaterlandes. In: Ergießungen Deutschen Gefühles in Gesängen und Liedern bey den Ereignissen dieser Zeit. [Heidelberg]. S. 130-131. 
Schopenhauer, Johanna (1813): Erinnerungen von einer Reise in den Jahren 1803, 1804 und 1805. Bd. 1. Rudolfstadt.

Weber, Ernst (1991): Lyrik der Befreiungskriege (1812-1815). Gesellschaftspolitische Meinungsund Willensbildung durch die Literatur. Stuttgart.

Wilson, Eric G. (2003): The Spiritual History of Ice: Romanticism, Science and the Imagination. New York/Basingstoke.

Woźniakowski, Jacek (1974): Góry niewzruszone. O różnych wyobrażeniach przyrody w dziejach nowożytnej kultury europejskiej. Warszawa.

Zelle, Carsten (1989): Schönheit und Erhabenheit. Der Anfang doppelter Ästhetik bei Boileau, Dennis, Bodmer und Breitinger. In: Christine Pries (Hrsg.): Das Erhabene. Zwischen Grenzerfahrung und Größenwahn. Weinheim. S. 55-73.

Ziehen, Eduard (1922): Die deutsche Schweizerbegeisterung in den Jahren 1750-1815. Frankfurt am Main. 